\title{
ERRATUM TO: THE WIGNER-VILLE DISTRIBUTION ASSOCIATED WITH THE QUATERNION OFFSET LINEAR CANONICAL TRANSFORM
}

\author{
Y. EL HAOUI \\ École Normale Supérieure (ENS-Meknès), Moulay Ismaïl University, 3104, Toulal, Meknès, \\ Morocco \\ e-mails: y.elhaoui@umi.ac.ma, youssefelhaoui@gmail.com
}

(Received May 3, 2021; revised September 27, 2021; accepted September 27, 2021)

Erratum to: Analysis Mathematica, 45 (2019), 787-802

DOI: $10.1007 / \mathrm{s} 10476-019-0007-0$

In [1, Lemma 3.2], the function $h$ should not depend on the variable $u$, and the lemma must be stated as follows (see [2]):

LEMma 3.2. The QOLCT of a signal $f \in L^{1}\left(\mathbb{R}^{2}, \mathbb{H}\right)$ can be reduced to the QFT

$$
\begin{gathered}
\mathcal{O}_{A_{1}, A_{2}}^{\lambda, \mu}\{f\}\left(u_{1}, u_{2}\right)=e^{\lambda\left[-\frac{1}{b_{1}} u_{1}\left(d_{1} \tau_{1}-b_{1} \eta_{1}\right)+\frac{d_{1}}{2 b_{1}}\left(u_{1}^{2}+\tau_{1}^{2}\right)\right]} \mathcal{F}^{\lambda, \mu}\{h\}\left(\frac{u_{1}}{b_{1}}, \frac{u_{2}}{b_{2}}\right) \\
\times e^{\mu\left[-\frac{1}{b_{2}} u_{2}\left(d_{2} \tau_{2}-b_{2} \eta_{2}\right)+\frac{d_{2}}{2 b_{2}}\left(u_{2}^{2}+\tau_{2}^{2}\right)\right]},
\end{gathered}
$$

with

$$
h(t)=\frac{1}{\sqrt{2 \pi \lambda b_{1}}} e^{\lambda\left(\frac{1}{b_{1}} t_{1} \tau_{1}+\frac{a_{1}}{2 b_{1}} t_{1}^{2}\right)} f(t) e^{\mu\left(\frac{1}{b_{2}} t_{2} \tau_{2}+\frac{a_{2}}{2 b_{2}} t_{2}^{2}\right)} \frac{1}{\sqrt{2 \pi \mu b_{2}}} .
$$

In [1, Definition 4.1], the definition of the WVD-QOLCT is correct for the case $b_{1}, b_{2} \neq 0$. However, for the three cases $b_{1}=0, b_{2} \neq 0, b_{1} \neq 0, b_{2}=0$ and $b_{1}=b_{2}=0$, one should not have the integration symbol, and the definition must be given as follows:

Key words and phrases: Wigner-Ville distribution, offset linear canonical transform, linear canonical transform, quaternionic transform, Heisenberg uncertainty, Poisson summation formula, Lieb's inequality.

Mathematics Subject Classification: $42 \mathrm{~A} 32$. 


$$
\begin{gathered}
\mathcal{W}_{f, g}^{A_{1}, A_{2}}(t, u)= \\
\begin{cases}\int_{\mathbb{R}^{2}} K_{A_{1}}^{\lambda}\left(s_{1}, u_{1}\right) f\left(t+\frac{s}{2}\right) \bar{g}\left(t-\frac{s}{2}\right) K_{A_{2}}^{\mu}\left(s_{2}, u_{2}\right) d s, & b_{1}, b_{2} \neq 0, \\
\sqrt{d_{1}} e^{\lambda\left(\frac{c_{1} d_{1}}{2}\left(u_{1}-\tau_{1}\right)^{2}+u_{1} \tau_{1}\right)} f\left(t_{1}+\frac{d_{1}\left(u_{1}-\tau_{1}\right)}{2}, t_{2}+\frac{s_{2}}{2}\right) & b_{1}=0, b_{2} \neq 0, \\
\times \bar{g}\left(t_{1}-\frac{d_{1}\left(u_{1}-\tau_{1}\right)}{2}, t_{2}-\frac{s_{2}}{2}\right) K_{A_{2}}^{\mu}\left(s_{2}, u_{2}\right) d s, & \\
\sqrt{d_{2}} K_{A_{1}}^{\lambda}\left(s_{1}, u_{1}\right) f\left(t_{1}+\frac{s_{1}}{2}, t_{2}+\frac{d_{2}\left(u_{2}-\tau_{2}\right)}{2}\right) & b_{1} \neq 0, b_{2}=0, \\
\times \bar{g}\left(t_{1}-\frac{s_{1}}{2}, t_{2}-\frac{d_{2}\left(u_{2}-\tau_{2}\right)}{2}\right) e^{\mu\left(\frac{c_{2} d_{2}}{2}\left(u_{2}-\tau_{2}\right)^{2}+u_{2} \tau_{2}\right)} & \\
\left.\left.\sqrt{d_{1} d_{2}} e^{\lambda\left(\frac{c_{1} d_{1}}{2}\left(u_{1}-\tau_{1}\right)^{2}+u_{1} \tau_{1}\right)} f\left(t_{1}+\frac{d_{1}\left(u_{1}-\tau_{1}\right.}{2}\right), t_{2}+\frac{d_{2}\left(u_{2}-\tau_{2}\right.}{2}\right)\right) & \\
\left.\left.\times \bar{g}\left(t_{1}-\frac{d_{1}\left(u_{1}-\tau_{1}\right.}{2}\right), t_{2}-\frac{d_{2}\left(u_{2}-\tau_{2}\right.}{2}\right)\right) e^{\mu\left(\frac{c_{2} d_{2}}{2}\left(u_{2}-\tau_{2}\right)^{2}+u_{2} \tau_{2}\right)}, & b_{1}=b_{2}=0,\end{cases}
\end{gathered}
$$

In [1, Remark 4.2], and in all the rest of the paper, the function $h$ must be noted $h_{f, g, t}(s)$, that is, the variable $t$ is fixed. Thus relation (4.1) will be in the form

$$
\mathcal{W}_{f, g}^{A_{1}, A_{2}}(t, u)=\mathcal{O}_{A_{1}, A_{2}}^{\lambda, \mu}\left\{h_{f, g, t}\right\}(u) .
$$

In [1, Lemma 4.3], the function $k_{f, g}$ must not depend on the variable $u$ on which $\mathcal{F}^{\lambda, \mu}$ depends. The correction of this lemma is as follows:

Lemma 4.3. We have

$$
\begin{aligned}
\mathcal{W}_{f, g}^{A_{1}, A_{2}}(t, u)= & e^{\lambda\left[-\frac{1}{b_{1}} u_{1}\left(d_{1} \tau_{1}-b_{1} \eta_{1}\right)+\frac{d_{1}}{2 b_{1}}\left(u_{1}^{2}+\tau_{1}^{2}\right)\right]} \mathcal{F}^{\lambda, \mu}\left\{k_{f, g, t}\right\}\left(\frac{u_{1}}{b_{1}}, \frac{u_{2}}{b_{2}}\right) \\
& \times e^{\mu\left[-\frac{1}{b_{2}} u_{2}\left(d_{2} \tau_{2}-b_{2} \eta_{2}\right)+\frac{d_{2}}{2 b_{2}}\left(u_{2}^{2}+\tau_{2}^{2}\right)\right]},
\end{aligned}
$$

where

$$
k_{f, g, t}(s)=\frac{1}{\sqrt{2 \pi \lambda b_{1}}} e^{\lambda\left(\frac{1}{b_{1}} s_{1} \tau_{1}+\frac{a_{1}}{2 b_{1}} s_{1}^{2}\right)} h_{f, g, t}(s) e^{\mu\left(\frac{1}{b_{2}} s_{2} \tau_{2}+\frac{a_{2}}{2 b_{2}} s_{2}^{2}\right)} \frac{1}{\sqrt{2 \pi \mu b_{2}}} .
$$

In the statement of [1, Theorem 4.9], the function $f\left(t+\frac{s}{2}\right) \bar{g}\left(t-\frac{s}{2}\right)$ should be written $f\left(t+\frac{s+k}{2}\right) \bar{g}\left(t-\frac{s+k}{2}\right)$ and the theorem can be stated in an improved form as follows:

Theorem 4.9. Let $f, g \in L^{2}\left(\mathbb{R}^{2}, \mathbb{H}\right)$. Then we have

$$
\sum_{\left(k_{1}, k_{2}\right) \in \mathbb{Z}^{2}} e^{\frac{i}{b_{1}} k_{1} \tau_{1}+i \frac{a_{1}}{2 b_{1}} k_{1}^{2}} f\left(t+\frac{k}{2}\right) \bar{g}\left(t-\frac{k}{2}\right) e^{\frac{j}{b_{2}} k_{2} \tau_{2}+j \frac{a_{2}}{2 b_{2}} k_{2}^{2}}
$$




$$
\begin{gathered}
=\sqrt{2 \pi i b_{1}}\left[\sum_{\left(k_{1}, k_{2}\right) \in \mathbb{Z}^{2}} e^{2 \pi i k_{1}\left(d_{1} \tau_{1}-b_{1} \eta_{1}\right)-i \frac{d_{1}}{2 b_{1}}\left(4 \pi^{2} b_{1}^{2} k_{1}^{2}+\tau_{1}^{2}\right)}\right. \\
\times \mathcal{W}_{f, g}^{A_{1}, A_{2}}\left(t,\left(2 \pi b_{1} k_{1}, 2 \pi b_{2} k_{2}\right)\right) \\
\left.\times e^{2 \pi j k_{2} s_{2}} e^{2 \pi j k_{2}\left(d_{2} \tau_{2}-b_{2} \eta_{2}\right)-j \frac{d_{2}}{2 b_{2}}\left(4 \pi^{2} b_{2}^{2} k_{2}^{2}+\tau_{2}^{2}\right)}\right] \sqrt{2 \pi j b_{2}} .
\end{gathered}
$$

ProOF. Let $\omega_{f, g, t}(s)=e^{i \frac{1}{b_{1}} s_{1} \tau_{1}+i \frac{a_{1}}{2 b_{1}} s_{1}^{2}} f\left(t+\frac{s}{2}\right) \bar{g}\left(t-\frac{s}{2}\right) e^{j \frac{1}{b_{2}} s_{2} \tau_{2}+j \frac{a_{2}}{2 b_{2}} s_{2}^{2}}$.

As $f, g \in L^{2}\left(\mathbb{R}^{2}, \mathbb{H}\right)$, by Hölder's inequality we have $\omega_{f, g, t} \in L^{1}\left(\mathbb{R}^{2}, \mathbb{H}\right)$. Then by Proposition 4.8 one has

$$
\begin{gathered}
\sum_{\left(k_{1}, k_{2}\right) \in \mathbb{Z}^{2}} \omega_{f, g, t}\left(s_{1}+k_{1}, s_{2}+k_{2}\right) \\
=\sum_{\left(k_{1}, k_{2}\right) \in \mathbb{Z}^{2}} e^{2 \pi i k_{1} s_{1}} \mathcal{F}^{i, j}\left\{\omega_{f, g, t}\right\}\left(2 \pi k_{1}, 2 \pi k_{2}\right) e^{2 \pi j k_{2} s_{2}} .
\end{gathered}
$$

Applying (4.2) leads to

$$
\begin{gathered}
\sum_{\left(k_{1}, k_{2}\right) \in \mathbb{Z}^{2}} e^{\frac{i}{b_{1}}\left(s_{1}+k_{1}\right) \tau_{1}+i \frac{a_{1}}{2 b 1}\left(s_{1}+k_{1}\right)^{2}} \\
\times f\left(t+\frac{s+k}{2}\right) \bar{g}\left(t-\frac{s+k}{2}\right) e^{\frac{j}{b_{2}}\left(s_{2}+k_{2}\right) \tau_{2}+j \frac{a_{2}}{2 b_{2}}\left(s_{2}+k_{2}\right)^{2}} \\
=\sqrt{2 \pi i b_{1}} \sum_{\left(k_{1}, k_{2}\right) \in \mathbb{Z}^{2}} e^{2 \pi i k_{1} s_{1}} e^{i\left[2 \pi k_{1}\left(d_{1} \tau_{1}-b_{1} \eta_{1}\right)-\frac{d_{1}}{2 b_{1}}\left(4 \pi^{2} b_{1}^{2} k_{1}^{2}+\tau_{1}^{2}\right)\right]}
\end{gathered}
$$

$\times \mathcal{W}_{f, g}^{A_{1}, A_{2}}\left(t,\left(2 \pi b_{1} k_{1}, 2 \pi b_{2} k_{2}\right)\right) e^{j\left[2 \pi k_{2}\left(d_{2} \tau_{2}-b_{2} \eta_{2}\right)-\frac{d_{2}}{2 b_{2}}\left(4 \pi^{2} k_{2}^{2} b_{2}^{2}+\tau_{2}^{2}\right)\right]} e^{2 \pi j k_{2} s_{2}} \sqrt{2 \pi j b_{2}}$.

Therefore, we get the result by evaluating $\left(s_{1}, s_{2}\right)=(0,0)$.

[1, Lemma 4.12] must be corrected as follows:

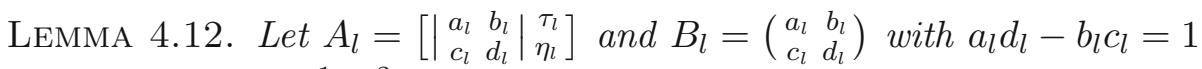
for $l=1,2$. For $f \in L^{1}\left(\mathbb{R}^{2}, \mathbb{H}\right)$, we have the relation

$$
\begin{gathered}
\mathcal{O}_{A_{1}, A_{2}}^{\lambda, \mu}\{f(t)\}(u)=e^{\lambda\left(-2 u_{1}\left(d_{1} \tau_{1}-b_{1} \eta_{1}\right)+d_{1} \tau_{1}^{2}\right) \frac{1}{2 b_{1}}} \\
\times \mathcal{L}_{B_{1}, B_{2}}\left\{e^{\lambda \frac{\tau_{1}}{b_{1}} t_{1}} f(t) e^{\mu \frac{\tau_{2}}{b_{2}} t_{2}}\right\}(u) e^{\mu\left(-2 u_{2}\left(d_{2} \tau_{2}-b_{2} \eta_{2}\right)+d_{2} \tau_{2}^{2}\right) \frac{1}{2 b_{2}}} .
\end{gathered}
$$


The proof of [1, Theorem 4.11] starts as follows:

By equation (4.14) we have

$$
\begin{gathered}
\left(\int_{\mathbb{R}^{2}}\left|\mathcal{W}_{f, g}^{A_{1}, A_{2}}(t, u)\right|_{Q}^{p} d u\right)^{\frac{1}{p}}=\left(\int_{\mathbb{R}^{2}}\left|\mathcal{O}_{A_{1}, A_{2}}^{\lambda, \mu}\left\{h_{f, g, t}(s)\right\}(u)\right|_{Q}^{p} d u\right)^{\frac{1}{p}} \\
=\left(\int_{\mathbb{R}^{2}}\left|\mathcal{L}_{B_{1}, B_{2}}^{\lambda, \mu}\left\{e^{\lambda \frac{\tau_{1}}{b_{1}} s_{1}} h_{f, g, t}(s) e^{\mu \frac{\tau_{2}}{b_{2}} s_{2}}\right\}(u)\right|_{Q}^{p} d u\right)^{\frac{1}{p}} \\
\leq \frac{\left|b_{1} b_{2}\right|^{\frac{-1}{2}+\frac{1}{p}}}{2 \pi} \underbrace{\left(\int_{\mathbb{R}^{2}}\left|e^{\lambda \frac{\tau_{1}}{b_{1}} s_{1}} f\left(t+\frac{s}{2}\right) \bar{g}\left(t-\frac{s}{2}\right) e^{\mu \frac{\tau_{2}}{b_{2}} s_{2}}\right|_{Q}^{q} d s\right)^{\frac{1}{q}}}_{=\left(\int_{\mathbb{R}^{2}}\left|f\left(t+\frac{s}{2}\right) \bar{g}\left(t-\frac{s}{2}\right)\right|_{Q}^{q} d s\right)^{\frac{1}{q}}} .
\end{gathered}
$$

The rest of the proof is valid.

Acknowledgement. The author is grateful to the editor and the two anonymous referees, whose insightful comments improved the paper immensely.

\section{References}

[1] M. El Kassimi, Y. El Haoui and S. Fahlaoui, The Wigner-Ville distribution associated with the quaternion offset linear canonical transform, Anal. Math., 45 (2019), 787-802.

[2] Y. El Haoui and E. Hitzer, Generalized uncertainty principles associated with the quaternionic offset linear canonical transform, Complex Var. Elliptic Equ., https://doi.org/10.1080/17476933.2021.1916919. 